\title{
Nível de atividade física habitual em portadores e não portadores de neuropatia diabética
}

\author{
Habitual physical activity level in bearers and non-bearers of diabetic \\ neuropathy
}

Carlos Gabriel de Lade', João Carlos Bouzas Marins', Luciana Moreira Lima', Maicon Rodrigues Albuquerque', Robson Bonoto Teixeira', Janice Sepúlveda Reis², Paulo Roberto dos Santos Amorim

\section{Resumo}

$\mathrm{O}$ aumento das atividades físicas habituais (AFH), como a caminhada e as consequentes contrações musculares delas oriundas, pode ser considerado uma estratégia de auxílio do controle metabólico em diabéticos. Apesar disso, complicações como a polineuropatia periférica simétrica distal (PSD) em pessoas com diabetes podem contribuir diretamente para a redução dos níveis da AFH. Com isso, o objetivo do presente estudo foi avaliar e comparar variáveis sanguíneas e o nível de AFH estimado por número de passos em diabéticos portadores e não portadores de PSD. Vinte e sete diabéticos, sendo 14 não portadores e 13 portadores de PSD atendidos no Centro Hiperdia de Viçosa, foram avaliados quanto ao nível de atividade física habitual estimada por número de passos/dia através de pedômetros. Cada participante utilizou o equipamento durante sete dias e os dados relacionados a cinco dias de semana e dois dias do final de semana foram analisados. Além disso, foram submetidos à avaliações antropométricas e bioquímicas. Não foram observadas diferenças significativas nas variáveis antropométricas e bioquímicas entre os grupos. O grupo sem PSD apresentou médias de passos em 7 dias, nos dias de semana e dias de final de semana estatisticamente superiores ao grupo com PSD $(\mathrm{p}<0,05)$. Apesar do grupo sem PSD ser mais ativo, os dois grupos apresentam baixos níveis de AFH estimada por número de passos/dia, evidenciando a necessidade de elaboração de programas educacionais de atividades físicas específicos para ambas as populações.

\section{Palavras-chave}

Diabetes mellitus; Neuropatias diabéticas; Atividade física.

\begin{abstract}
The increase of habitual physical activity (HPA), such as walks and the consequent muscular contraction originated, can be considered a strategy to improve metabolic control in diabetics. Nevertheless, complications as the peripheral symmetrical distal polyneuropathy (PSD) in people with diabetes can contribute directly for HPA reduction. The aim of this study was evaluate and compare blood variables and the HPA level estimated by the number of steps in diabetics bearers and non-bearers of PSD. Twenty seven diabetics, being 14 non-bearers and 13 bearers of PSD treated in the Hiperdia Center of Viçosa, were evaluated in terms of the HPA level estimated by the number of steps/day through pedometers. Each participant used the equipment during seven days and the data of five weekdays and 2 weekend days were analyzed. Furthermore, they were submitted through a anthropometric and biochemical evaluation. Significant differences were not observed in the anthropometric and biochemical variables between groups. The group without PSD presented a mean of steps in 7 days, in the weekdays and weekend statistically superior to the group with PSD ( $\mathrm{p}<0.05)$. Although the group without PSD was more active, both groups presented low levels of HPA estimated by the number of steps/day, evidencing the necessity of elaborating educational programs of physical activity for both populations.
\end{abstract}

\section{Keywords}

Diabetes Mellitus; Diabetics Neuropathies; Physical Activity.

\section{Introdução}

A morbidade e mortalidade associadas ao diabetes

1 Universidade Federal de Viçosa, Departamento de Educação Física, Viçosa, Minas Gerais, Brasil.

2 Coordenadora do Ambulatório de Diabetes Tipo 1 da Santa Casa de Belo Horizonte, Belo Horizonte, Minas Gerais, Brasil. são significativas e crescentes ${ }^{1}$. O envelhecimento da população, o aumento das taxas de obesidade, inatividade física e o crescimento populacional são características esperadas para alavancar a prevalência do diabetes mellitus (DM). Segundo estimativas atuais, 382 milhões de pessoas são portadoras de DM e a previsão é que esse número atinja mais de 590 milhões em 
$2035^{2}$. Ainda, segundo a International Diabetes Federation, o Brasil possui hoje quase 12 milhões de pessoas com DM, ocupando o quarto lugar no ranking mundial'.

Dentre as diversas comorbidades associadas a um controle glicêmico inadequado, destaca-se a neuropatia como a mais frequente das complicações microvasculares do DM, com mais de 50\% das pessoas com diabetes tipo 2 (DM 2) apresentando evidências de neuropatia distal. As neuropatias são caracterizadas pela perda progressiva de fibras nervosas, tanto do sistema nervoso autonômico quanto somático, com fisiopatologia ainda não bem compreendida ${ }^{3}$.

Hiperglicemia sustentada, inflamação, hereditariedade e tabagismo parecem ser fatores de risco que contribuem para o desenvolvimento de neuropatias. A polineuropatia periférica simétrica distal (PSD), a mais comum neuropatia dolorosa, pode levar a déficits motores e sensoriais, com disfunções relacionadas à mobilidade, alterações na característica da marcha ${ }^{4}$ e deficiências no equilíbrio ${ }^{5}$, sendo uma das principais causas de incapacidade devido a dor, ulcerações, amputações e quedas ${ }^{6}$.

Estudos demonstraram que a taxa de mortalidade por todas as causas e por doenças cardiovasculares são menores em pacientes com DM 2 ativos fisicamente 7 . Entretanto, apesar do exercício físico ser considerado fundamental para o tratamento do DM 2, uma porção considerável dessa população não é fisicamente ativa ${ }^{8}$.

O estímulo ao aumento das atividades físicas habituais (AFH), como caminhar para o trabalho ou subir escadas, pode ser uma alternativa para elevar o gasto energético diário da população em geral ${ }^{9}$. De acordo com Tudor-Locke et al. ${ }^{10}$, o nível de atividade física de um indivíduo pode ser classificado de acordo com a média de passos diários, sendo considerado ativo aquele que atinge média de passos diária maior ou igual a 10000 passos. Em contrapartida, um indivíduo é considerado sedentário quando essa média é inferior a 5000 passos. Caminhar o mínimo de 10000 passos diários tem sido recomendado para a promoção da saúde e do bem estar ${ }^{11}$.

Apesar da recomendação de 10000 passos/dia ser o mais reconhecido, essa diretriz pode tornar-se irreal para pacientes com $\mathrm{DM}^{12}$. Estudos populacionais apontam uma frequência média de 7000 a 13000 passos diários em adultos de 20 a 50 anos aparentemente saudáveis. Já na população com DM, estudos apontaram média de 6291 passos/dia ${ }^{13}$. Apesar da manutenção de um estilo de vida ativo ser considerado uma importante estratégia de tratamento não medicamentoso do DM e de complicações associadas ${ }^{1}$, a avaliação do nível de $\mathrm{AFH}$ em pacientes diabéticos atendidos pelo sistema público de saúde não é comum na prática clínica. Com isso, o objetivo do presente estudo foi avaliar e comparar os parâmetros sanguíneos e o nível de atividades física diário através da contagem de passos em pacientes com DM 2 portadores e não portadores de PSD atendidos no Centro Hiperdia de Viçosa/MG.

\section{Métodos}

Realizou-se um estudo descritivo, quantitativo e de corte transversal, desenvolvido como parte do projeto "Avaliação e Tratamento de Diabéticos e Hipertensos Atendidos pelo Centro Hiperdia de Viçosa”, uma parceria da Universidade Federal de Viçosa (UFV) com o Programa Hiperdia Minas.

\section{Sujeitos}

A amostra do estudo foi composta por 27 sujeitos portadores de DM 2, sendo 13 pacientes portadores de PSD (10 homens e 3 mulheres) e 14 pacientes não portadores de PSD (5 homens e 9 mulheres), pertencentes a uma faixa etária de 45 a 68 
anos e que atenderam a Resolução SES no 2.606 de sete de Dezembro de 2010 em que se estabelece os critérios de encaminhamento para os Centros de Referência Integrados Viva Vida e Hiperdia Minas. Como critérios de exclusão foram considerados sujeitos portadores de diabetes mellitus tipo 1, retinopatia grave, doença arterial periférica, neuropatia autonômica, claudicação intermitente, funções musculares ou esqueléticas prejudicadas, doenças psiquiátricas, doenças coronarianas e que apresentavam algum tipo de amputação e ulcerações graves nos pés.

O estudo foi aprovado pelo Comitê de Ética em Pesquisa com Seres Humanos da UFV sob o número de registro 37759114.7.0000.5153 na Plataforma Brasil e CAAE 28144814000005153, atendendo assim às normas da Legislação Brasileira (Resolução 466/12) sobre estudos com seres humanos. Todos os participantes selecionados para o estudo eram atendidos pelo Centro Hiperdia de Viçosa/MG, foram informados a respeito da metodologia e dos objetivos do estudo e assinaram o Termo de Consentimento Livre e Esclarecido.

\section{Instrumentos}

A coleta de dados incluiu dados antropométricos e clínicos dos pacientes tais como: sexo, idade, massa corporal, estatura, índice de massa corporal (IMC), perímetro do abdome, presença de hipertensão arterial sistêmica (HAS), glicemia de jejum, glicohemoglobina (HbA1c), glicemia média estimada, colesterol total, frações e triglicérides. As amostras bioquímicas foram coletadas e analisadas por laboratório credenciado pelo Sistema Único de Saúde, sob encaminhamento e autorização dos médicos responsáveis do Centro Hiperdia de Viçosa. A presença ou não de HAS foi considerada de acordo com o diagnóstico realizado pelos cardiologistas do Centro Hiperdia de Viçosa. Essa informação estava contida nos prontuários de cada paciente.

A massa corporal foi medida utilizando-se uma balança Mercy ${ }^{\circledR}$ (modelo LC 200, Brasil, 2010), com escala variando de 1 a 200 quilogramas e com 50 gramas de precisão. A estatura foi avaliada através de estadiômetro Welmy ${ }^{\circledR}$ (modelo R110, Brasil, 2009) com escala variando de 0,8 a 2,00 metros com 1 milímetro de precisão. O IMC foi calculado através da divisão da massa corporal $(\mathrm{Kg})$ pela estatura $(\mathrm{m})^{2}$. O perímetro do abdome foi mensurado utilizando-se fita métrica retrátil e flexível Proximus ${ }^{\circledR}$ (Rio de Janeiro, Brasil, 2013), com escala de 0 a 200 centímetros e precisão de 1 milímetro, utilizando-se a cicatriz umbilical como ponto de referência. As avaliações antropométricas foram realizadas no Hiperdia em um local reservado, feito por um profissional de Educação Física. O número de passos foi avaliado através de pedômetros (Digiwalker ${ }^{\circledR}, \mathrm{CW}-700$, Yamax Corporation, Tokyo, Japan), com método semelhante ao utilizado por Oliveira et al. ${ }^{9}$.

\section{Procedimentos}

O diagnóstico para PSD foi realizado através de screening realizado por uma enfermeira responsável pelo setor de tratamento de pé diabético do Centro Hiperdia de Viçosa/MG, seguindo as recomendações propostas pelo Consenso Internacional sobre Pé Diabético ${ }^{14}$, sendo que a seleção dos voluntários ocorreu a partir do diagnóstico de PSD presente nos prontuários.

O nível de AFH estimado ocorreu através da contagem do número de passos durante 8 dias consecutivos, incluindo os dias de final de semana. Todas as orientações dos sujeitos selecionados ocorreram nas dependências do Centro Hiperdia de Viçosa/MG e compreendiam instruções quanto ao manuseio do equipamento, cuidados, segurança e a não alterarem sua rotina diária. Foram instruídos a utilizar o equipa- 
mento ao acordar e retirá-lo somente para tomar banho e dormir, além da retirada do equipamento ao deslocarem-se utilizando ônibus, motocicletas e bicicletas.

Uma ficha de anotações foi entregue a cada sujeito contendo 8 campos em branco representando os dias de utilização do pedômetro. $\mathrm{O}$ equipamento deveria ser fixado do lado direito do quadril, no cinto ou na própria roupa. Ao se deitarem para dormir, os participantes foram orientados a abrir o equipamento e anotar o número mostrado na tela. Os dados dos participantes analfabetos ou que apresentaram dificuldade na compreensão da dinâmica do estudo foram registrados por parentes, que foram orientados quanto a dinâmica do estudo. No dia seguinte ao completar os 8 dias de utilização do pedômetro, os participantes encaminhavam-se ao Hiperdia para a devolução dos equipamentos.

Após a entrega dos pedômetros, ocorreu a conferência dos valores através da memória do equipamento e das anotações nas fichas. O resultado do primeiro dia foi descartado para minimizar o efeito Hawthorne, onde o processo de avaliação altera o fenômeno avaliado?.

Os sujeitos foram classificados de acordo com Tudor-Locke et al..$^{10}$ : sedentários (<5000 passos/dia), menos ativos (5000-7499 passos/dia), pouco ativos (7500-9999 passos/dia), ativos (10000-12499 passos/dia) e altamente ativos ( $\geq 12500$ passos/dia).

\section{Análise Estatística}

O tamanho mínimo da amostra foi definido utilizando-se o coeficiente de variação observado neste estudo para a medida dos passos diários (44\%), a partir da própria amostra avaliada, considerando-se $25 \%$ de variação em torno da média. Com isso, o número mínimo de 13 participantes em cada grupo foi considerado para que fosse possível verificar diferenças estatísticas com um nível de 5\% de significância ${ }^{15}$.

Foi realizado teste de Shapiro-Wilk para verificar a normalidade dos dados. Para a comparações inter grupos foram utilizados o teste $t$ independente para os dados que apresentaram distribuição normal e o teste de Mann-Whitney para os dados que não apresentaram distribuição normal. Nas comparações intra grupos, o teste t pareado foi utilizado para os dados com distribuição normal, enquanto que para comparações dos dados com distribuição não normal, o teste de Wilcoxon foi utilizado. Para verificar o grau de correlação entre a idade e a média de passos em 7 dias foi utilizado o coeficiente de correlação de Spearman. Foi considerado o nível de significância $\mathrm{p}<0,05$. Todas as análises foram realizadas utilizando-se o software SPSS (SPSS Inc. ${ }^{\circledR}$, versão 20 ).

\section{Resultados}

Inicialmente os grupo avaliados foram compostos por um total de 38 sujeitos, sendo 20 no grupo sem PSD e 18 no grupo com PSD. Como os pacientes atendidos pelo Centro Hiperdia de Viçosa apresentam baixo Índice de Desenvolvimento Humano (IDH), como baixa escolaridade, alguns pacientes apresentaram dificuldade na compreensão correta dos procedimentos necessários para a utilização e anotação dos dados dos pedômetros. Além disso, alguns pacientes retornaram para a entrega dos equipamento fora do prazo estipulado. Com isso, ocorreu uma perda de resultados de 5 voluntários do grupo com PSD e de 6 voluntários do grupo sem PSD.

Como demonstrado pela Tabela 1, a amostra selecionada apresentou características homogêneas, com diferença significativa apenas para a média de idade. A média de idade os sujeitos com PSD $(62,0 \pm 2,6)$ foi maior que a dos sujeitos sem 
$\operatorname{PSD}(52,0 \pm 2,6)$, sendo essa diferença estatisticamente significativa $(p=0,01)$, representando um tamanho do efeito $r=0,49$. Apesar de uma menor média de idade apresentada pelo grupo sem PSD, a análise de correlação realizada entre a idade e a média de passos/dia em 7 dias não apresentou-se estatisticamente significativa $(p=0,056)$.

TABELA 1 - Características da amostra segmentada em portadores e não portadores de PSD.

\begin{tabular}{|c|c|c|c|c|c|}
\hline \multirow{3}{*}{$\begin{array}{l}\text { Parâmetros } \\
\text { Sexo }\end{array}$} & \multirow{2}{*}{\multicolumn{2}{|c|}{$\frac{\text { Diabéticos sem PSD }}{(n=14)}$}} & \multirow{2}{*}{\multicolumn{2}{|c|}{$\begin{array}{c}\text { Diabéticos com PSD } \\
\qquad(n=13)\end{array}$}} & \multirow{4}{*}{$p$} \\
\hline & & & & & \\
\hline & Masculino & Feminino & Masculino & Feminino & \\
\hline & $5(64 \%)$ & $9(36 \%)$ & $3(77 \%)$ & $10(23 \%)$ & \\
\hline Idade (anos) & \multicolumn{2}{|c|}{$52 \pm 10^{*}$} & \multicolumn{2}{|c|}{$62 \pm 10^{*}$} & $0,01^{\#}$ \\
\hline Massa corporal (Kg) & \multicolumn{2}{|c|}{$84 \pm 22$} & \multicolumn{2}{|c|}{$74 \pm 12$} & $0,24^{ \pm}$ \\
\hline Estatura $(\mathrm{cm})$ & \multicolumn{2}{|c|}{$160 \pm 9$} & \multicolumn{2}{|c|}{$160 \pm 7$} & $0,95^{\#}$ \\
\hline $\mathrm{IMC}\left(\mathrm{Kg} / \mathrm{m}^{2}\right)$ & \multicolumn{2}{|c|}{$32,9 \pm 8.3$} & \multicolumn{2}{|c|}{$28,7 \pm 3.5$} & $0,24^{ \pm}$ \\
\hline Circunferência Abdominal (cm) & \multicolumn{2}{|c|}{$110 \pm 20$} & $105 \pm 10$ & $0,73^{f}$ & \\
\hline \multirow[t]{2}{*}{ Hipertensão Arterial } & Sim & Não & Sim & Não & \\
\hline & $8(57 \%)$ & $6(43 \%)$ & $10(77 \%)$ & $3(23 \%)$ & \\
\hline
\end{tabular}

Resultados das variáveis contínuas apresentadas em média e desvio padrão; n=tamanho da amostra; \#=teste t independente; $£=$ teste de Mann Whitney $U ;{ }^{*}=$ diferença significativa $p<0,05$.

Os resultados referentes aos parâmetros bioquímicos dos sujeitos do estudo são apresentados na Tabela 2, onde verifica-se que não houve diferença significativa em nenhum dos parâmetros avaliados.

TABELA 2 - Parâmetros bioquímicos dos pacientes portadores e não portadores de PSD.

\begin{tabular}{lccc}
\hline \multicolumn{1}{c}{ Parâmetros } & $\begin{array}{c}\text { Diabéticos sem PSD } \\
(\mathrm{n}=14)\end{array}$ & $\begin{array}{c}\text { Diabéticos com PSD } \\
(\mathrm{n}=13)\end{array}$ & $\mathrm{p}$ \\
\hline Glicemia de jejum (mg/dL) & $185 \pm 91$ & $213 \pm 86$ & $0,41^{\#}$ \\
\hline Hemoglobina A1c (\%) & $9 \pm 2$ & $10 \pm 2$ & $0,34^{\#}$ \\
\hline $\begin{array}{l}\text { Glicemiamédia Estimada (mg/ } \\
\text { dL) }\end{array}$ & $210 \pm 57$ & $230 \pm 51$ & $0,34^{\#}$ \\
\hline Colesterol total (mg/dL) & $175 \pm 35$ & $187 \pm 52$ & $0,49^{\#}$ \\
\hline LDL (mg/dL) & $103 \pm 32$ & $111 \pm 44$ & $0,71^{\ddagger}$ \\
\hline VLDL (mg/dL) & $29 \pm 22$ & $34 \pm 20$ & $0,40^{\ddagger}$ \\
\hline HDL (mg/dL) & $48 \pm 10$ & $42 \pm 7$ & $0,08^{\#}$ \\
\hline Triglicérides (mg/dL) & $124 \pm 61$ & $182 \pm 86$ & $0,05^{\ddagger}$ \\
\hline
\end{tabular}

Resultados apresentados em média e desvio padrão; n=tamanho da amostra; \#=teste t independente; $£=$ teste de Mann Whitney U; PSD = polineuropatia periférica simétrica distal; nível de significância p<0,05.

Além do cálculo da média geral de passos para os dois grupos, o número total de passos avaliados em 7 dias foi segmentado em número de passos em dias de semana (DS) e fim de semana (FS) (Tabela 3). Para a média geral de passos avaliada em 7 dias, o grupo sem PSD apresentou média superior $(70,5 \pm 28,0)$ que o grupo portador de PSD $(46,6 \pm 24,0)$, com diferença significativa $(p=0,02)$ e tamanho $r=0,42$. O mesmo ocorreu para a média de passos nos DS, com o grupo sem PSD apresentando média superior $(97,3 \pm 38,7)$ ao grupo com PSD $(61,7 \pm 33,6)$, com diferença estatística $(p=0,001)$ e $r=0,9$. Nas médias de passos de FS, o grupo sem PSD apresentou média superior $(66,8 \pm 41,7)$ quando comparados com o grupo com PSD $(38,4 \pm 20,8)$, com diferença significativa $(p=0,004)$ e $r=0,58$. 
TABELA 3 - Diferenças entre os resultados do número de passos entre portadores e não portadores de PSD.

\begin{tabular}{lccc}
\hline \multirow{2}{*}{ Número de passos } & Diabéticos sem PSD & Diabéticos com PSD & \multirow{2}{*}{$p$} \\
\cline { 2 - 3 } & $(n=14)$ & $(n=13)$ & \\
\hline Média Geral & $70,5 \pm 280^{*}$ & $46,6 \pm 24,0^{*}$ & $0,02^{£}$ \\
\hline Média Dias de Semana (DS) & $97,3 \pm 38,7^{*}$ & $61,7 \pm 33,6^{*}$ & $0,001^{£}$ \\
\hline Média Fim de Semana (FS) & $66,8 \pm 41,7^{*}$ & $38,4 \pm 20,8^{*}$ & $0,004^{£}$ \\
\hline
\end{tabular}

Resultados apresentados em média e desvio padrão; $n=$ tamanho da amostra; $£=$ teste de Mann Whitney U; PSD = polineuropatia periférica simétrica distal; * =diferença significativa $\mathrm{p}<0,05$.

Ao analisarmos as médias do número de passos divididos em dias de semana (DS) e final de semana (FS), os resultados apresentados pela Tabela 4 demonstram diferenças significativas nas comparações intra grupos. Para o grupo sem PSD, observou-se média de passos superior nos DS $(97,3 \pm 38,7)$ do que nos FS $(66,8 \pm 41,7)$, apresentando diferença significativa $(p=0,008)$ e $r=0,51$, o mesmo ocorrendo com o grupo com PSD, que apresentou média nos DS $(61,7 \pm 33,6)$ superior à média nos FS $(38,4 \pm 20,8)$, também com diferença significativa $(p=0,006)$ e $r=0,53$.

TABELA 4 - Comparação intra grupos entre a média de passos nos DS.

\begin{tabular}{lccc}
\hline Número de passos & Média de passos nos DS & Média de passos nos FS & $p$ \\
\hline Diabéticos sem PSD $(n=14)$ & $9732 \pm 3876^{\mathrm{a}}$ & $6686 \pm 4175^{\mathrm{a}}$ & $0,008^{£}$ \\
\hline Diabéticos com PSD $(\mathrm{n}=13)$ & $6175 \pm 3364^{\mathrm{b}}$ & $3845 \pm 2081^{\mathrm{b}}$ & $0,006^{£}$ \\
\hline
\end{tabular}

Resultados apresentados em média e desvio padrão; $n=$ tamanho da amostra; $£=$ teste de Wilcoxon; PSD = polineuropatia periférica simétrica distal; $a ; b=$ diferença significativa $\mathrm{p}<0,05$.

A Figura 1 representa a média geral de passos em 7 dias para cada sujeito. Apenas 3 indivíduos não portadores de PSD atingiram a média de 10000 passos diários, enquanto que 4 sujeitos não atingiram média de 5000 passos diários. Já para os sujeitos portadores de PSD, apenas 1 atingiu a meta de 10000 passos diários, enquanto que 10 indivíduos apresentaram média inferior a 5000 passos.

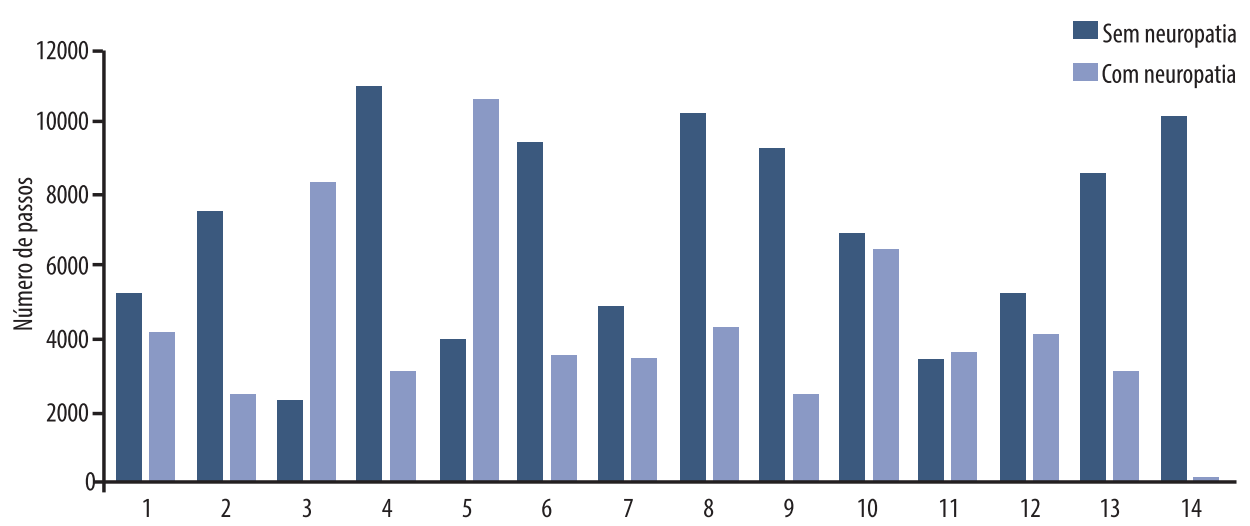

FIGURA 1 - Média geral de passos por pessoa.

\section{Discussão}

Objetivamos verificar o nível de AFH, representados pelos números de passos por dia, em indivíduos diabéticos divididos em grupos com e sem PSD. Ambos os grupos apresentaram resultados aquém das recomendações de 10000 passos dias, com o grupo sem PSD apresentando-se mais ativo, com média de 7050 passos/dia, do que o grupo com PSD, com a média de 4663 passos/dia. 
A média geral de passos em 7 dias para não portadores PSD (7050 passos/dia) foi superior aos resultados encontrados em indivíduos com DM apresentados por Richardson et al. ${ }^{16}$ (4596 passos/dia), contudo semelhante aos valores encontrados por Araiza et al. ${ }^{17}$ (7220 passos/dia). Em relação aos portadores de PSD (4663 passos/dia), o resultado foi semelhante aos resultados encontrado por Armstrong et al. ${ }^{18}$ (4548 passos/dia). De acordo com Van Sloten et al. ${ }^{19}$, a associação entre a PSD com a redução da força muscular, perda de equilíbrio, marcha prejudicada e risco aumentado de quedas pode afetar a manutenção de um estilo de vida ativo, estando associada à reduções de 1800 a 1950 passos por dia. Na comparação das médias entre os grupos avaliados, as limitações impostas pela presença da doença podem ter contribuído pela menor média de passos apresentada pelo grupo com PSD.

Najafi et al. ${ }^{20}$ demonstraram que sujeitos com PSD apresentaram apenas $6 \%$ do tempo avaliado em atividades de deslocamento (caminhando), o que corrobora com a baixa média de passos encontrada no grupo com PSD. A contagem diária de passos é amplamente aceita por pesquisadores e profissionais de saúde para a avaliação, acompanhamento e prescrição de atividades físicas ${ }^{21}$, sendo que a contagem de passos/dia representa 62 e $67 \%$ da variabilidade no tempo gasto com atividades físicas em mulheres e homens, respectivamente ${ }^{22}$. A média de passos/ dia de 7000 a 8000 passos aproxima-se das recomendações para a prática regular de atividades físicas de, no mínimo, 30 minutos/dia ou 150 minutos/semana ${ }^{21}$, demonstrado que o grupo sem PSD encontra-se dentro dessas recomendações, enquanto o grupo com PSD atinge um nível de atividade física diária bem abaixo do recomendado.

Corroborando com essa análise, a Figura 1 demonstra que no grupo com PSD, 10 sujeitos apresentaram média de passos/dia menor que 5000 passos, demonstrando uma elevada taxa de sedentarismo nesse grupo, enquanto no grupo sem a doença, 4 sujeitos não atingiram essa média. Em relação a classificação "ativo" (10000-12499 passos/dia), 3 sujeitos sem PSD e apenas 1 sujeito com a doença atingiram essa classificação. Os resultados demonstram um baixo nível de atividade físicas diárias em ambos os grupos, o que pode explicar parcialmente a ausência de diferenças dos parâmetros relacionados ao metabolismo glicêmico e nos elevados valores apresentados.

Indivíduos portadores de DM devem evitar dois dias ou mais sem atividades físicas e, além disso, reduzir o tempo despendido em atividades sedentárias ${ }^{23}$. A redução da média de passos nos FS em ambos os grupos (Tabela 4) demonstra claramente a diminuição das atividades físicas habituais com o consequente aumento de atividades sedentárias.

Ambos os grupos demonstraram-se homogêneos, com diferenças estatisticamente significativas apenas nas médias de idade. Os danos aos nervos podem ocorrer a qualquer momento, mas o risco é aumentado com a idade e pelo tempo de instalação do diabetes e, de acordo com Van Schie ${ }^{4}$, mais de $50 \%$ de sujeitos com diabetes acima de 60 anos de idade apresentam evidências de PSD, o que pode explicar a diferença de idade entre os grupos. Contudo, apesar de uma menor média de idade apresentadas pelo grupo sem PSD, os resultados da correlação entre a idade e número de passos não apresentou-se estatisticamente significativo, minimizando a influência da idade sobre número de passos/dia.

Não foram verificadas diferenças estatisticamente significativas entre os grupos em nenhum dos parâmetros sanguíneos avaliados. A média dos valores tanto da glicemia de jejum quanto da hemoglobina A1c para os dois grupos demonstraram um controle metabólico ineficaz de acordo com a American Diabetes Asso- 
ciation $^{23}$, que estabelece valores toleráveis até $130 \mathrm{mg} / \mathrm{dL}$ e menores que $7 \%$ para glicemia de jejum e $\mathrm{HbA1c}$, respectivamente. O controle glicêmico ineficaz, com hiperglicemias sustentadas nos anos anteriores ao diagnóstico, pode ser considerado um dos principais fatores de risco para o desenvolvimento da $\mathrm{PSD}^{3}$.

Independentemente da significância estatística não verificada os resultados das análises sanguíneas dos pacientes com PSD demonstraram valores mais elevados nos parâmetros avaliados, e é importante considerar que verificamos a diferença de $1 \%$ nos valores absolutos na comparação das médias de HbA1c entre os grupos. Apesar de não apresentarem diferenças significativas, a diferença de $1 \%$ em números absolutos nos valores de HbA1c representa o aumento de 15 a $20 \%$ do risco de eventos cardiovasculares e de $37 \%$ no risco de complicações microvasculares ${ }^{24}$. Esses resultados são semelhantes aos apresentados por Van Dyck et al. ${ }^{12}$, ao compararem grupos de diabéticos mais e menos ativos também através da pedometria, onde verificaram valores superiores no grupo menos ativo, porém sem diferenças significativas, em relação ao grupo mais ativo nos mesmo parâmetros sanguíneos do presente estudo.

Além da prática de atividades físicas regulares, como a caminhada, ser um componente importante no tratamento do DM, auxiliando o controle glicêmico eficaz e reduzindo riscos cardiovasculares ${ }^{23,25,26}$, também é uma alternativa para retardar ou impedir a progressão da PSD, teoricamente agindo sobre a modulação do processo inflamatório e no controle glicêmico ${ }^{27}$. Apesar das evidências apontarem para a importância de um estilo de vida fisicamente ativo para o controle eficaz do $\mathrm{DM}^{23}$, existe um número limitado de evidências da associação entre atividade física e a PSD ${ }^{1}$. Entretanto, os resultados dos estudos de Balducci et al..$^{28}$, Kluding et al. ${ }^{29} \mathrm{e}$ Tuttle et al. ${ }^{30}$ demonstraram uma relação inversa entre a prática regular de atividade física e a evolução da PSD.

Consequências importantes relacionadas à PSD podem limitar um estilo de vida ativo $^{19}$, além da prática de atividades físicas com a sustentação da massa corporal frequentemente serem desencorajadas para essa população devido ao risco aumentado de quedas, ulcerações e amputações ${ }^{25}$. Em contrapartida, evidências atuais sugerem que atividades moderadas com sustentação da massa corporal podem diminuir o risco de ulcerações quando comparados com indivíduos menos $\operatorname{ativos}^{1,23,25,30}$, principalmente se houver o mínimo de variação no padrão de atividades físicas diárias.

Um programa de caminhadas progressivas pode preservar os músculos das extremidades inferiores, aumentando a tolerância do tecido plantar ao estresse, tornando-os menos propensos a desenvolver ulcerações ${ }^{20}$, além da manutenção da mobilidade e da aptidão física geral ${ }^{30}$. Além disso, 150 minutos de atividade físicas moderadas por semana foram relatados como auxílio no controle de neuropatias mais brandas ${ }^{23}$, tornado a caminhada, com suas características acessíveis a qualquer população, um instrumento importante no controle do diabetes e complicações associadas ${ }^{30}$.

Como limitações do presente estudo, destaca-se a redução do tamanho amostral pela não compreensão dos procedimentos metodológicos e perda de dados por alguns participantes, além das limitações impostas pelos critérios de exclusão. Outra limitação a destacar é a utilização de pedômetros na avaliação de atividades físicas habituais. Apesar do registro do número diário de passos, esses equipamentos são incapazes de mensurar a frequência, a duração e a intensidade das atividades. Porém, devido ao seu baixo custo e fácil aceitação por parte dos participantes, frequentemente têm sido utilizados e são considerados um instrumento 
adequado de avaliação das atividades físicas habituais. Sugerimos a replicação do estudo com a utilização de acelerômetros para uma análise mais criteriosa do nível de $\mathrm{AFH}$, já que esses equipamentos são capazes de fornecer dados a respeito da frequência, duração e intensidade dessas atividades ${ }^{9}$.

Os resultados do presente estudo nos permite concluir que a comparação do nível de atividade física habitual entre dois grupos de diabéticos demonstrou que ambos são insuficientemente ativos, e ainda permitiu verificar que o grupo com PSD apresentou médias significativamente inferiores nas comparações realizadas nos dias de semana, fim de semanae total dos dias avaliados em relação ao grupo sem PSD. Independentemente dessas diferenças na AFH não foram verificadas diferenças estatisticamente significativas em nenhum dos parâmetros sanguíneos analisados.

\section{Contribuição dos autores}

Carlos G. de Lade participou do planejamento, coleta e análise dos dados e redação do manuscrito. Robson B. Teixeira contribuiu com a coleta e análise do dados e correção do texto. João C. B. Marins e Luciana M. Lima participaram da correção, revisão do texto e orientação na coleta de dados. Maicon R. Albuquerque participou da análise dos dados e revisão do texto. Janice S. Reis participou da revisão e correção do texto. Paulo R. S. Amorim orientou o planejamento, coleta dos dados, revisão e correção do texto.

\section{Agradecimentos}

Secretaria Estadual de Saúde de Minas Gerais. Secretaria Municipal de Saúde de Viçosa/MG.

\section{Financiamento}

Fundação de Amparo à Pesquisa do Estado de Minas Gerais - FAPEMIG.

\section{Referências}

1. Loprinzi PD, Hager KK, Ramulu, PY. Physical activity, glycemic control, and diabetic peripheral neuropathy: A national sample. J Diabetes Complications. 2014;28(1):17-21.

2. International Diabetes Federation. IDF Diabetes Atlas 2013. 6th Ed.

3. Boulton AJM. Management of Diabetic Peripheral Neuropathy. Clinical Diabetes. 2005;23(1): 9-15.

4. Van Schie CHM. Neuropathy: mobility and quality of life. Diabetes Metab Res Rev. 2008;24 Suppl 1: 45-51.

5. Bonnet C, Carello C, Turvey MT. Diabetes and postural stability: review and hypotheses. J Mot Behav. 2009;41(2):172-90.

6. Pop-Busui R, Lu J, Lopes N, Jones TL, BARI 2D Investigators. Prevalence of diabetic peripheral neuropathy and relation to glycemic control therapies at baseline in the BARI 2D cohort. J Peripher Nerv Syst. 2009;14(1):1-13.

7. Wei M, Gibbons LW, Kampert JB, Nichaman MZ, Blair SN. Low cardiorespiratory fitness and physical inactivity as predictors of mortality in men with type 2 diabetes. Ann Intern Med. 2000;132(8):605-11.

8. Tudor-Locke C, Bell RC, Myers AM, Harris SB, Ecclestone NA, Lauzon N, et al. Controlled outcome evaluation of the First Step Program: a daily physical activity intervention for individuals with type II diabetes. Int J Obes. 2004;28(1):113-19.

9. Oliveira MB, Moura BP, Marins JCB, Juvêncio JF, Amorim PRS. Nível de atividade física habitual e laboral estimada por pedômetros. Rev Bras Ativ Fís Saúde. 2011;16(3):188-92.

10. Tudor-Locke C, Hatano Y, Pangrazi RP, Kang M. Revisiting "How many steps are enough?” Med Sci Sports Exerc. 2008;40(7):537-43.

11. Ananian CD, Soroush A, Ainsworth B, Belyea M, Walker J, Poortvliet E, et al. Trajectories and predictors of steps in a worksite intervention: ASUK-Step. Health Behav Policy Rev. 2015;2(1):46-61. 
12. Van Dick D, De Greef K, Deforche B, Ruige J, Bouckaert J, Tudor-Locke CE, et al. The relationship between changes in steps/day and health outcomes after a pedometerbased physical activity intervention with telephone support in type 2 diabetes patients. Health Educ Res. 2013;28(3):539-45.

13. Tudor-Locke C, Washington TL, Hart TL. Expected values for steps/day in special populations. Prev Med. 2009;49(1):3-11.

14. Grupo de trabalho internacional sobre pé diabético. Consenso internacional sobre pé diabético. Brasília: Secretaria de Estado de Saúde do Distrito Federal; 2001.

15. Hulley SB, Cummings SR. Estimating sample size and power. In: Designing Clinical Research. Baltimore, Md: Williams and Wilkins. 1988:148, Appendlix 13A, 215.

16. Richardson CR, Mehari KS, McIntyre LG, Janney AW, Fortlage LA, Sen A, et al. A randomized trial comparing structured and lifestyle goals in an internet-mediated walking program for people with type 2 diabetes. Int J Behav Nutr Phys Act. 2007;4(59):1-11.

17. Araiza P, Hewes H, Gashetewa C, Vella CA, Burge MR. Efficacy of a pedometer-based physical activity program on parameters of diabetes control in type 2 diabetes mellitus. Metabolism. 2006;55(10):1382-7.

18. Armstrong DG, Abu-Rumman PL, Nixon BP, Boulton AJ. Continuous activity monitoring in persons at high risk for diabetes-related lower-extremity amputation. J Am Podiatr Med Assoc. 2001;91(9):451-5.

19. Van Sloten TT, Savelberg HH, Duimel-Peeters IG, Meijer K, Henry RM, Stehouwer CD, et al. Peripheral neuropathy, decreased muscle strength and obesity are strongly associated with walking in persons with type 2 diabetes without manifest mobility limitations. Diabetes Res Clin Pract. 2011;91(1):32-9.

20. Najafi B, Crews RT, Wrobel JS. Importance of time spent standing for those at risk of diabetic foot ulceration. Diabetes Care. 2010;33(11):2448-50.

21. Tudor-Locke C, Craig CL, Thyfalt JP, Spence JC. A step-defined sedentary lifestyle index: $<5000$ steps/day. Appl Physiol Nutr Metab. 2013;38(2): 100-14.

22. Tudor-Locke C, Johnson WD, Katzmarzyk PT. Relationship between accelerometerdetermined steps/day and other accelerometer outputs in U.S. adults. J Phys Act Health. 2011;8(3):410-19.

23. American Diabetes Association (ADA). Standards of Medical Care in Diabetes-2015. Diabetes Care. 2015;38 (Suppl 1):S1-94.

24. Sigal RJ, Kenny GP, Boulé NG, Wells GA, Prud'homme D, Fortier M et al. Effects of aerobic training, resistance training, or both on glycemic control in type 2 diabetes. Ann Intern Med. 2007;147(6):357-69.

25. Kruse RL, Lemaster JW, Madsen RW. Fall and balance outcomes after an intervention to promote leg strength, balance, and walking in people with diabetic peripheral neuropathy: "Feet First" Randomized Controlled Trial. Phys Ther. 2010;90(11):1568-79.

26. Van Schie CHM, Noordhof EL, Busch-Westbroek TE, Beelen A, Nollet F. Assessment of physical activity in people with diabetes and peripheral neuropathy. Brief Report. Diabetes Res Clin Pract. 2011;92:9-11.

27. Loprinzi PD, Cardinal B, Crespo C, Brodowicz G, Andersen R, Sullivan E, et al. Objectively measured physical activity and C-reactive protein: National Health and Nutrition Examination Survey 2003-2004. Scand J Med Sci Sports. 2013;23(2):164-70.

28. Balducci S, Iacobellis G, Parisi L, Di Biase N, Calandriello E, Leonetti F, et al. Exercise training can modify the natural history of diabetic peripheral neuropathy. J Diabetes Complications. 2006;20(4):216-23.

29. Kluding PM, Pasnoor M, Singh R, Jernigan S, Farmer K, Rucker J, Sharma NK, et al. The effect of exercise on neuropathic symptoms, nerve function, and cutaneous innervation in people with diabetic peripheral neuropathy. J Diabetes Complications. 2012;26(5):424-9.

30. Tuttle LJ, Hastings MK, Mueller MJ. A Moderate-Intensity Weight-Bearing Exercise Program for a Person With Type 2 Diabetes and Peripheral Neuropathy. Phys Ther. 2012;92(1):133-41.

ENDEREÇO PARA

CORRESPONDÊNCIA CARLOS GABRIEL DE LADE lade.gab@gmail.com
Universidade Federal de Viçosa.

Departamento de Educação Física.

Av. PH Rolfs, s/n. Campus Universitário,

Viçosa-MG. 36570-900.

Tel: (32) 98835-2726 